\title{
Impact of Social Isolation on People with Dementia and Their Family Caregivers
}

Lílian Viana dos Santos Azevedo ${ }^{\mathrm{a}}$, Ismael Luis Calandri ${ }^{\mathrm{b}}$, Andrea Slachevsky ${ }^{\mathrm{c}, \mathrm{d}, \mathrm{e}, \mathrm{f}}$, Héctor Gastón Graviottog ${ }^{\mathrm{g}}$, Maria Carolina Santos Vieira ${ }^{\mathrm{h}, \mathrm{i}}$, Caíssa Bezerra de Andrade ${ }^{\mathrm{a}}$, Adriana Peredo Rossetti ${ }^{\text {h,i }}$, Alana Barroso Generosoi, Karoline Carvalho Carmona ${ }^{\mathrm{a}}$, Ludmilla Aparecida Cardoso Pinto ${ }^{\mathrm{a}}$, Marcos Sorbara ${ }^{\mathrm{g}}$, Alejandra Pinto ${ }^{\mathrm{d}}$, Tania Guajardo ${ }^{\mathrm{d}}$, Loreto Olavarria $^{\mathrm{d}}$, Daniela Thumala ${ }^{\mathrm{c}, \mathrm{j}}$, Lucía Crivelli ${ }^{\mathrm{b}}$, Ludmila Vivas ${ }^{\mathrm{b}}$, Ricardo Francisco Allegrib ${ }^{\mathrm{b}}$, Maira Tonidandel Barbosa ${ }^{\mathrm{a}, \mathrm{h}}$, Cecilia M. Serrano ${ }^{\mathrm{g}}$, Claudia Miranda-Castillok, and Paulo Caramelli ${ }^{\mathrm{a}, *}$

${ }^{a}$ Behavioral and Cognitive Research Group, Department of Internal Medicine, Faculdade de Medicina, Universidade Federal de Minas Gerais, Belo Horizonte (MG), Brazil

${ }^{\mathrm{b}}$ Department of Cognitive Neurology, Neuropsychology and Neuropsychiatry, FLENI, Buenos Aires, Argentina ${ }^{\mathrm{c}}$ Geroscience Center for Brain Health and Metabolism (GERO), Santiago, Chile

${ }^{\mathrm{d}}$ Memory and Neuropsychiatric Clinic (CMYN) Neurology Department, Hospital del Salvador and Faculty of Medicine, University of Chile, Santiago, Chile

${ }^{\mathrm{e}}$ Neuropsychology and Clinical Neuroscience Laboratory (LANNEC), Physiopathology Department - ICBM, Neurocience and East Neuroscience Departments, Faculty of Medicine, University of Chile, Santiago, Chile

${ }^{\mathrm{f}}$ Servicio de Neurología, Departamento de Medicina, Clínica Alemana-Universidad del Desarrollo, Santiago, Chile

${ }^{\mathrm{g}}$ Cognitive Neurology and Neuropsychology Department, César Milstein Hospital, Buenos Aires, Argentina

${ }^{\mathrm{h}}$ Faculdade Ciências Médicas de Minas Gerais/FELUMA, Belo Horizonte (MG), Brazil

${ }^{\mathrm{i}}$ Geriatric Medicine - Rede Mater Dei de Saúde e AURUS Ensino e Pesquisa do Envelhecimento, Belo Horizonte (MG), Brazil

${ }^{\mathrm{j} D e p a r t m e n t ~ o f ~ P s y c h o l o g y, ~ S o c i a l ~ S c i e n c e s ~ F a c u l t y, ~ U n i v e r s i t y ~ o f ~ C h i l e, ~ S a n t i a g o, ~ C h i l e ~}$

${ }^{\mathrm{k}}$ Faculty of Nursing, Universidad Andres Bello, Santiago, Chile

${ }^{1}$ Millennium Institute for Research in Depression and Personality, Santiago, Chile

\footnotetext{
Abstract. their family caregivers. dementia and in their caregivers.

${ }^{*}$ Correspondence to: Paulo Caramelli, MD, PhD, Faculdade de Medicina, Universidade Federal de Minas Gerais, Av. Prof. Alfredo Balena, 190 - sala 246, 30130-100, Belo Horizonte (MG), Brazil. E-mail: caramelli@ufmg.br.
}

Background: People with dementia and their family caregivers may face a great burden through social isolation due to the COVID-19 pandemic, which can be manifested as various behavioral and clinical symptoms.

Objective: To investigate the impacts of social isolation due to the COVID-19 pandemic on individuals with dementia and

Methods: Two semi-structured questionnaires were applied via telephone to family caregivers of people diagnosed with dementia in three cities in Argentina, Brazil, and Chile, in order to assess clinical and behavioral changes in people with 
Results: In general, 321 interviews were conducted. A significant decline in memory function has been reported among $53.0 \%$ of people with dementia. In addition, $31.2 \%$ of individuals with dementia felt sadder and $37.4 \%$ had increased anxiety symptoms. These symptoms of anxiety were greater in individuals with mild to moderate dementia, while symptoms of agitation were greater in individuals with severe dementia. Moreover, compulsive-obsessive behavior, hallucinations, increased forgetfulness, altered appetite, and increased difficulty in activities of daily living were reported more frequently among individuals with moderate to severe dementia. Caregivers reported feeling more tired and overwhelmed during this period and these symptoms were also influenced by the severity of dementia.

Conclusion: Social isolation during the COVID-19 pandemic triggered a series of negative behavioral repercussions, both for people with dementia and for their family caregivers in these three South American countries.

Keywords: Behavioral symptoms, caregiver, COVID-19, dementia, social isolation

\section{INTRODUCTION}

COVID-19, the disease caused by the severe acute respiratory syndrome coronavirus 2 (SARS-CoV-2), is currently the biggest health challenge worldwide. In Latin America, by the beginning of February 2021, there had been more than 19 million confirmed cases and more than 600,000 deaths [1].

Knowledge about the virus is still limited, but social isolation seems to be an effective strategy for controlling outbreaks of COVID-19 [2]. For this reason, physical distancing measures have been implemented in many countries, as a way to contain the speed of viral transmission, reduce the chances that healthcare systems could collapse and diminish mortality.

Social distancing can reduce disease transmission, which is important for controlling the spread of this virus. However, it can cause a series of clinical and psychological problems, since it increases the possibility that cognitive, cardiovascular, and autoimmune changes might develop, along with depression and anxiety [3-7].

People with dementia and their family caregivers, whose daily lives already entail the hardships that dementia imposes, may suffer even more through social isolation. People with dementia may find it difficult to understand and adapt to changes in their routines, as well as not remembering the preventive care that they need to take. They may therefore be at greater risk of contracting COVID-19 infection [8]. This scenario also has the potential to cause them to exhibit behavioral changes, which may lead to or exacerbate symptoms such as anxiety, agitation, aggressiveness, irritability, and sleep disorders, among others. Family caregivers may likewise be impacted by the changes in routine imposed through social isolation, which can result in greater burdens and negative repercussions on their mental health [9].

There is little published information about how people with dementia and their family caregivers are coping with the pandemic and isolation, particularly in relation to low- and middle-income countries. In just a few studies, especially from Europe, increased symptoms of agitation and anxiety among individuals diagnosed with dementia have been reported, in addition to an increased burden on family caregivers during this period [21, 22].

Data from around the world is beginning to emerge in the literature, but information remains very scarce, especially in relation to countries that have low investment in healthcare services. Therefore, there is a continuing need to evaluate how social isolation can affect the lives of individuals diagnosed with dementia, in order to develop possible assistance strategies in this period and in other contexts of confinement that may arise in the future.

\section{METHODS}

\section{Study design and subjects}

This was a cross-sectional study in which the impacts of social isolation due to the COVID-19 pandemic on individuals with dementia and their family caregivers was investigated. Participants were recruited from five outpatient clinics in South America: two in Argentina, two in Brazil, and one in Chile, from May to July 2020. Family caregivers of people with mild, moderate, or severe dementia, of any etiology and without any age or sex restrictions, were included in the study.

This study was approved by the research ethics committees of all participating centers and written 
informed consent was obtained from all participants. Each clinic was responsible for recruiting its participants and for sending out the consent form in advance via e-mail, with all the information about the study. The family caregivers were asked to read this information and those who freely agreed to participate sent back the signed statement, also by e-mail. Individuals diagnosed with mild cognitive impairment and caregivers who refused to participate or who did not sign the informed consent statement were excluded.

\section{Data collection and processing}

Data collection was performed by means of telephone interviews using two semi-structured questionnaires that had been prepared by the study investigators. Both questionnaires were answered by the family caregiver. One questionnaire related to the caregiver and the other to the individual with dementia.

The questionnaires focused on the sociodemographic, clinical, and behavioral characteristics of people with dementia and their family caregivers, namely, age, sex, education level, socioeconomic status (through appropriate instruments for each country), kinship, medical diagnosis, and severity of dementia. The possible behavioral and cognitive changes experienced during this period of physical distancing were also evaluated, such as anxiety, agitation, sedentary lifestyle, altered memory, sleep disorders, and caregiver burden, among others.

Family caregivers reported on whether they or their relatives with dementia had experienced any changes, by comparing their condition before and during social isolation. There were three answer options: yes, partially, or no. The questionnaires used for the patients and the caregivers are displayed as Supplementary Materials 1 and 2.

The severity of dementia was determined using the Functional Assessment Scale (FAST), which enables staging by observing patients' cognitive impairment and their degree of functional dependence. FAST has seven stages, ranging from 1 to 7 . In the present study, the following stages of dementia were considered: mild (stage 4), moderate (stage 6), and severe, from stage $6 \mathrm{a}$ (needs assistance to put on clothes) to stage $7 \mathrm{f}$ (loss of ability to maintain head support) [10].

The socioeconomic level was established through an economic evaluation criterion that stratifies families into six social classes (A, B1, B2, C1, C2, D-E), in which class $\mathrm{A}$ is the highest socioeconomic level and classes D-E are the lowest socioeconomic levels [11].

\section{Statistical analysis}

Descriptive statistical analyses were performed on all demographic data, clinical data, and behavioral characteristics. Descriptive summaries were presented as means or medians (with standard deviation, SD) for continuous variables and as proportions for categorical variables. The Kolmogorov-Smirnov and Shapiro-Wilk tests were used to test the distribution of continuous variables. The one-way ANOVA test was used to compare quantitative variables between groups. Pearson's chi-square test or Fisher's exact test were used to compare the frequencies of categorical variables, as appropriate.

To test the independence of symptomatic variables and dementia severity, the Cochran-Mantel-Haenszel method was used in a model adjusted for age strata (in quartiles) and socioeconomic level. Due to the large number of independent tests, the BenjaminiHochberg method was used to correct for multiple comparisons.

A value of $p<0.05$ was considered significant. The statistical analyses were performed using the R software, version 3.6.1, with the tidy verse, psych, and Table 1 packages.

\section{RESULTS}

\section{Participants' characteristics}

Overall, 321 interviews were carried out with family caregivers in the three countries: 108 in Argentina, 111 in Brazil, and 102 in Chile. The individuals diagnosed with dementia had an average age of 77.2 years [SD $=9.3$ ] and $57.0 \%$ were female, while the family caregivers had an average age of 58.8 years $[\mathrm{SD}=15.3]$ and $78.8 \%$ were female. There was no statistical difference in relation to age or sex across the three countries, between the individuals with dementia or between the caregivers (Table 1).

The educational level of the people with dementia was highest in Argentina and lowest in Brazil. Regarding the severity of dementia, the participants were less impaired in Argentina than in the other two countries. Impairment was most severe in Brazil, while the distribution was more homogeneous in Chile (Table 1).

The educational level (in years) was higher among individuals with mild dementia and lower among individuals with severe dementia. In addition, socioeconomic status was also higher among individuals diagnosed with mild dementia (Table 2). 
Table 1

Sociodemographic and clinical characteristics of study participants in the three countries

\begin{tabular}{|c|c|c|c|c|c|}
\hline & Argentina $(N=108)$ & $\operatorname{Brazil}(N=111)$ & Chile $(N=102)$ & Global $(N=321)$ & $p$ \\
\hline \multicolumn{6}{|l|}{ People with dementia } \\
\hline \multicolumn{6}{|l|}{ Age } \\
\hline Mean (SD) & $77.4(8.25)$ & $78.0(10.1)$ & $76.2(9.54)$ & $77.2(9.35)$ & \multirow[t]{2}{*}{0.373} \\
\hline Median [Min, Max] & $79.0[34.0,91.0]$ & $79.0[50.0,100]$ & $77.0[43.0,94.0]$ & $79.0[34.0,100]$ & \\
\hline \multicolumn{6}{|l|}{ Sex } \\
\hline Female & $63(58.3 \%)$ & $63(56.8 \%)$ & $57(55.9 \%)$ & $183(57.0 \%)$ & 0.936 \\
\hline \multicolumn{6}{|l|}{ Education (y) } \\
\hline Mean (SD) & $11.6(4.73)$ & $7.63(6.08)$ & $9.69(4.81)$ & $9.62(5.49)$ & \multirow[t]{2}{*}{$<0.001$} \\
\hline Median [Min, Max] & $12.0[2.00,18.0]$ & $4.00[0,25.0]$ & $10.0[0,19.0]$ & $10.0[0,25.0]$ & \\
\hline \multicolumn{6}{|l|}{ Dementia severity } \\
\hline Mild & $96(88.9 \%)$ & $35(31.5 \%)$ & $30(29.4 \%)$ & $161(50.2 \%)$ & \multirow{3}{*}{$<0.001$} \\
\hline Moderate & $8(7.4 \%)$ & $28(25.2 \%)$ & $38(37.3 \%)$ & $74(23.1 \%)$ & \\
\hline Severe & $4(3.7 \%)$ & $48(43.2 \%)$ & $34(33.3 \%)$ & $86(26.8 \%)$ & \\
\hline \multicolumn{6}{|l|}{ Socioeconomic level } \\
\hline A & $22(20.4 \%)$ & $13(11.7 \%)$ & $4(3.9 \%)$ & $39(12.1 \%)$ & \multirow[t]{4}{*}{$<0.001$} \\
\hline $\mathrm{B}$ & $46(42.6 \%)$ & $37(33.3 \%)$ & $32(31.4 \%)$ & $115(35.8 \%)$ & \\
\hline $\mathrm{C}$ & $37(34.3 \%)$ & $42(37.8 \%)$ & $59(57.8 \%)$ & $138(43.0 \%)$ & \\
\hline D-E & $2(1.9 \%)$ & $18(16.2 \%)$ & $3(2.9 \%)$ & $23(7.2 \%)$ & \\
\hline \multicolumn{6}{|l|}{ Caregivers } \\
\hline \multicolumn{6}{|l|}{ Age } \\
\hline Mean (SD) & $60.5(16.8)$ & $57.1(13.9)$ & $59.1(15.2)$ & $58.8(15.3)$ & \multirow[t]{2}{*}{0.251} \\
\hline Median [Min, Max] & $63.0[22.0,87.0]$ & $56.0[22.0,86.0]$ & $60.0[23.0,88.0]$ & $59.0[22.0,88.0]$ & \\
\hline \multicolumn{6}{|l|}{ Sex } \\
\hline Female & $79(73.1 \%)$ & $90(81.1 \%)$ & $84(82.4 \%)$ & $253(78.8 \%)$ & 0.258 \\
\hline \multicolumn{6}{|l|}{ Education } \\
\hline Mean (SD) & $13.6(3.79)$ & $11.3(4.71)$ & $13.2(3.97)$ & $12.6(4.31)$ & \multirow[t]{2}{*}{$<0.001$} \\
\hline Median [Min, Max] & $12.0[3.00,20.0]$ & $11.0[0,23.0]$ & $14.0[2.00,21.0]$ & $12.0[0,23.0]$ & \\
\hline
\end{tabular}

Table 2

Sociodemographic and clinical characteristics of study participants in relation to severity of dementia

\begin{tabular}{lcccc}
\hline & \multicolumn{3}{c}{ Dementia severity } & Adjusted $p^{2}$ \\
\cline { 2 - 4 } & Mild & $\begin{array}{c}\text { Moderate } \\
N=74^{1}\end{array}$ & $\begin{array}{c}\text { Severe } \\
N=86^{1}\end{array}$ & \\
\hline $\begin{array}{l}\text { People with dementia } \\
\text { Age }\end{array}$ & $79(73,83)$ & $78(73,82)$ & $80(71,85)$ & 0.5 \\
Sex & $94(58 \%)$ & $43(58 \%)$ & $46(53 \%)$ & 0.7 \\
$\quad$ Female & $12.0(7.0,15.0)$ & $8.5(4.0,14.0)$ & $6.0(4.0,11.0)$ & $<0.001$ \\
Education (y) & $29(18 \%)$ & $4(5.5 \%)$ & $6(7.1 \%)$ & \\
Socioeconomic level & $67(42 \%)$ & $25(34 \%)$ & $23(27 \%)$ & 0.001 \\
A & $53(34 \%)$ & $37(51 \%)$ & $48(57 \%)$ & \\
B & $9(5.7 \%)$ & $7(9.6 \%)$ & $7(8.3 \%)$ & \\
C & & & & \\
D-E & $58(47,73)$ & $55(46,65)$ & $62(53,72)$ & 0.023 \\
Caregivers & & & & \\
Age & $117(73 \%)$ & $68(92 \%)$ & $68(79 \%)$ & 0.005 \\
Sex & $13.0(11.0,17.0)$ & $13.0(11.0,16.0)$ & $12.0(8.0,15.0)$ & 0.002 \\
$\quad$ Female & & & \\
Education (y) & & & \\
\hline
\end{tabular}

${ }^{1}$ Median (IQR); n (\%) ${ }^{2}$ Kruskal-Wallis rank sum test; Pearson's Chi-squared test.

\section{Social isolation and clinical and behavioral} symptoms of people with dementia

Regarding behavioral symptoms that emerged among people with dementia due to social isolation, the impact of isolation differed according to the aspect that was probed: $31.2 \%$ felt sadder, $16.2 \%$ felt more lonely, $37.4 \%$ were more anxious, $23.1 \%$ had greater agitation and $16.8 \%$ became more aggressive, $19 \%$ had delusions, $15.9 \%$ presented hallucinations, and $18.1 \%$ presented some type of obsessive behavior (Table 3).

Cognitive and functional changes in people with dementia were also frequently reported. A significant 
Table 3

Behavioral, cognitive and functional changes in people with dementia due to social isolation

\begin{tabular}{|c|c|c|c|c|c|}
\hline & $\begin{array}{l}\text { Argentina } \\
(N=108)\end{array}$ & $\begin{array}{c}\text { Brazil } \\
(N=111)\end{array}$ & $\begin{array}{c}\text { Chile } \\
(N=102)\end{array}$ & $\begin{array}{c}\text { Total } \\
(N=321)\end{array}$ & Adjusted $p$ \\
\hline \multicolumn{6}{|l|}{ Sadder } \\
\hline No & $62(57.4 \%)$ & $65(58.6 \%)$ & $42(41.2 \%)$ & $169(52.6 \%)$ & \multirow[t]{3}{*}{0.010} \\
\hline Partially & $18(16.7 \%)$ & $6(5.4 \%)$ & $25(24.5 \%)$ & $49(15.3 \%)$ & \\
\hline Yes & $27(25.0 \%)$ & $40(36.0 \%)$ & $33(32.4 \%)$ & $100(31.2 \%)$ & \\
\hline \multicolumn{6}{|l|}{ More lonely } \\
\hline No & $76(70.4 \%)$ & $91(82.0 \%)$ & $69(67.6 \%)$ & $236(73.5 \%)$ & \multirow[t]{3}{*}{0.226} \\
\hline Partially & $16(14.8 \%)$ & $4(3.6 \%)$ & $10(9.8 \%)$ & $30(9.3 \%)$ & \\
\hline Yes & $14(13.0 \%)$ & $16(14.4 \%)$ & $22(21.6 \%)$ & $52(16.2 \%)$ & \\
\hline \multicolumn{6}{|c|}{ More anxious } \\
\hline No & $52(48.1 \%)$ & $64(57.7 \%)$ & $41(40.2 \%)$ & $157(48.9 \%)$ & \multirow[t]{3}{*}{0.082} \\
\hline Partially & $21(19.4 \%)$ & $6(5.4 \%)$ & $13(12.7 \%)$ & $40(12.5 \%)$ & \\
\hline Yes & $34(31.5 \%)$ & $41(36.9 \%)$ & $45(44.1 \%)$ & $120(37.4 \%)$ & \\
\hline \multicolumn{6}{|c|}{ More agitated } \\
\hline No & $81(75.0 \%)$ & $70(63.1 \%)$ & $63(61.8 \%)$ & $214(66.7 \%)$ & \multirow[t]{3}{*}{0.008} \\
\hline Partially & $15(13.9 \%)$ & $5(4.5 \%)$ & $11(10.8 \%)$ & $31(9.7 \%)$ & \\
\hline Yes & $11(10.2 \%)$ & $36(32.4 \%)$ & $27(26.5 \%)$ & $74(23.1 \%)$ & \\
\hline \multicolumn{6}{|c|}{ More aggressive } \\
\hline No & $87(80.6 \%)$ & $86(77.5 \%)$ & $55(53.9 \%)$ & $228(71.0 \%)$ & \multirow[t]{3}{*}{$<0.001$} \\
\hline Partially & $10(9.3 \%)$ & $6(5.4 \%)$ & $21(20.6 \%)$ & $37(11.5 \%)$ & \\
\hline Yes & $11(10.2 \%)$ & $18(16.2 \%)$ & $25(24.5 \%)$ & $54(16.8 \%)$ & \\
\hline \multicolumn{6}{|l|}{ Delusions } \\
\hline No & $98(90.7 \%)$ & $82(73.9 \%)$ & $68(66.7 \%)$ & $248(77.3 \%)$ & \multirow[t]{3}{*}{0.002} \\
\hline Partially & $3(2.8 \%)$ & $2(1.8 \%)$ & $7(6.9 \%)$ & $12(3.7 \%)$ & \\
\hline Yes & $7(6.5 \%)$ & $27(24.3 \%)$ & $27(26.5 \%)$ & $61(19.0 \%)$ & \\
\hline \multicolumn{6}{|c|}{ Obsessive-compulsive behaviors } \\
\hline No & $97(89.8 \%)$ & $90(81.1 \%)$ & $60(58.8 \%)$ & $247(76.9 \%)$ & \multirow[t]{3}{*}{$<0.001$} \\
\hline Partially & $5(4.6 \%)$ & $2(1.8 \%)$ & $6(5.9 \%)$ & $13(4.0 \%)$ & \\
\hline Yes & $5(4.6 \%)$ & $18(16.2 \%)$ & $35(34.3 \%)$ & $58(18.1 \%)$ & \\
\hline \multicolumn{6}{|c|}{ Hallucinations } \\
\hline No & $100(92.6 \%)$ & $94(84.7 \%)$ & $64(62.7 \%)$ & $258(80.4 \%)$ & \multirow[t]{3}{*}{$<0.001$} \\
\hline Partially & $2(1.9 \%)$ & $1(0.9 \%)$ & $8(7.8 \%)$ & $11(3.4 \%)$ & \\
\hline Yes & $6(5.6 \%)$ & $16(14.4 \%)$ & $29(28.4 \%)$ & $51(15.9 \%)$ & \\
\hline \multicolumn{6}{|c|}{ Memory decline } \\
\hline No & $44(40.7 \%)$ & $54(48.6 \%)$ & $19(18.6 \%)$ & $117(36.4 \%)$ & \multirow[t]{3}{*}{$<0.001$} \\
\hline Partially & $21(19.4 \%)$ & $1(0.9 \%)$ & $7(6.9 \%)$ & $29(9.0 \%)$ & \\
\hline Yes & $42(38.9 \%)$ & $56(50.5 \%)$ & $72(70.6 \%)$ & $170(53.0 \%)$ & \\
\hline \multicolumn{6}{|c|}{ Appetite changes } \\
\hline No & $72(66.7 \%)$ & $77(69.4 \%)$ & $43(42.2 \%)$ & $192(59.8 \%)$ & \multirow[t]{3}{*}{$<0.001$} \\
\hline Partially & $10(9.3 \%)$ & $1(0.9 \%)$ & $2(2.0 \%)$ & $13(4.0 \%)$ & \\
\hline Yes & $26(24.1 \%)$ & $33(29.7 \%)$ & $54(52.9 \%)$ & $113(35.2 \%)$ & \\
\hline \multicolumn{6}{|c|}{ Impaired activities of daily living } \\
\hline No & $91(84.3 \%)$ & $74(66.7 \%)$ & $43(42.2 \%)$ & $208(64.8 \%)$ & \multirow[t]{3}{*}{$<0.001$} \\
\hline Partially & $6(5.6 \%)$ & $4(3.6 \%)$ & $9(8.8 \%)$ & $19(5.9 \%)$ & \\
\hline Yes & $11(10.2 \%)$ & $32(28.8 \%)$ & $48(47.1 \%)$ & $91(28.3 \%)$ & \\
\hline Sleep difficu & & & & & \\
\hline No & $77(71.3 \%)$ & $89(80.2 \%)$ & $57(55.9 \%)$ & $223(69.5 \%)$ & $<0.001$ \\
\hline Partially & $13(12.0 \%)$ & $0(0 \%)$ & $9(8.8 \%)$ & $22(6.9 \%)$ & \\
\hline Yes & $17(15.7 \%)$ & $21(18.9 \%)$ & $36(35.3 \%)$ & $74(23.1 \%)$ & \\
\hline
\end{tabular}

decline in memory function was observed in $53.0 \%$ of the participants and impaired performance in activities of daily living was reported in $28.3 \%$ of the cases. Moreover, sleep changes emerged in $23.1 \%$ of the individuals (Table 3 ).
Regarding the severity of dementia, we found that the symptoms of anxiety were greater in individuals with mild to moderate dementia, and the symptoms of agitation were greater in individuals with severe dementia (Table 4). 
Table 4

Behavioral, cognitive, and functional changes due to social isolation among people with dementia according to severity

\begin{tabular}{|c|c|c|c|c|}
\hline & & ementia sever & & Adjusted $p$ \\
\hline & $\begin{array}{c}\text { Mild } \\
N=161^{1}\end{array}$ & $\begin{array}{c}\text { Moderate } \\
N=74^{1}\end{array}$ & $\begin{array}{c}\text { Severe } \\
N=86^{1}\end{array}$ & \\
\hline Sadder & & & & \\
\hline No & $84(53 \%)$ & $42(58 \%)$ & $43(50 \%)$ & \\
\hline Partially & $27(17 \%)$ & $11(15 \%)$ & $11(13 \%)$ & 0.566 \\
\hline Yes & $48(30 \%)$ & $20(27 \%)$ & $32(37 \%)$ & \\
\hline Loner & & & & \\
\hline No & $116(73 \%)$ & $55(75 \%)$ & $65(76 \%)$ & \\
\hline Partially & $18(11 \%)$ & $7(9.6 \%)$ & $5(5.8 \%)$ & 0.731 \\
\hline Yes & $25(16 \%)$ & $11(15 \%)$ & $16(19 \%)$ & \\
\hline More anxiou & & & & \\
\hline No & $75(47 \%)$ & $32(44 \%)$ & $50(59 \%)$ & \\
\hline Partially & $31(19 \%)$ & $5(6.9 \%)$ & $4(4.7 \%)$ & 0.007 \\
\hline Yes & $54(34 \%)$ & $35(49 \%)$ & $31(36 \%)$ & \\
\hline More agitate & & & & \\
\hline No & $115(72 \%)$ & $49(67 \%)$ & $50(58 \%)$ & \\
\hline Partially & $18(11 \%)$ & $7(9.6 \%)$ & $6(7.0 \%)$ & 0.055 \\
\hline Yes & $27(17 \%)$ & $17(23 \%)$ & $30(35 \%)$ & \\
\hline More aggres & & & & \\
\hline No & $126(79 \%)$ & $49(67 \%)$ & $53(62 \%)$ & \\
\hline Partially & $13(8.1 \%)$ & $10(14 \%)$ & $14(16 \%)$ & 0.373 \\
\hline Yes & $21(13 \%)$ & $14(19 \%)$ & $19(22 \%)$ & \\
\hline Delusion & & & & \\
\hline No & $138(86 \%)$ & $48(65 \%)$ & $62(72 \%)$ & \\
\hline Partially & $6(3.7 \%)$ & $4(5.4 \%)$ & $2(2.3 \%)$ & 0.003 \\
\hline Yes & $17(11 \%)$ & $22(30 \%)$ & $22(26 \%)$ & \\
\hline Obsessive-ce & & & & \\
\hline No & $137(86 \%)$ & $43(59 \%)$ & $67(79 \%)$ & \\
\hline Partially & $6(3.8 \%)$ & $4(5.5 \%)$ & $3(3.5 \%)$ & $<0.001$ \\
\hline Yes & $17(11 \%)$ & $26(36 \%)$ & $15(18 \%)$ & \\
\hline Hallucinatio & & & & \\
\hline No & $143(89 \%)$ & $54(74 \%)$ & $61(71 \%)$ & \\
\hline Partially & $3(1.9 \%)$ & $2(2.7 \%)$ & $6(7.0 \%)$ & 0.009 \\
\hline Yes & $15(9.3 \%)$ & $17(23 \%)$ & $19(22 \%)$ & \\
\hline Forgetting $\mathrm{m}$ & & & & \\
\hline No & $64(40 \%)$ & $21(30 \%)$ & $32(38 \%)$ & \\
\hline Partially & $25(16 \%)$ & $2(2.8 \%)$ & $2(2.4 \%)$ & 0.002 \\
\hline Yes & $71(44 \%)$ & $48(68 \%)$ & $51(60 \%)$ & \\
\hline Appetite cha & & & & \\
\hline No & $101(63 \%)$ & $39(54 \%)$ & $52(60 \%)$ & \\
\hline Partially & $12(7.5 \%)$ & $0(0 \%)$ & $1(1.2 \%)$ & 0.006 \\
\hline Yes & $47(29.0 \%)$ & $33(46.0 \%)$ & $33(38.0 \%)$ & \\
\hline Impaired act & & & & \\
\hline of daily livin & & & & \\
\hline No & $132(82 \%)$ & $37(51 \%)$ & $39(46 \%)$ & \\
\hline Partially & $13(8.1 \%)$ & $3(4.2 \%)$ & $3(3.5 \%)$ & $<0.001$ \\
\hline Yes & $16(9.9 \%)$ & $32(44.0 \%)$ & $43(51.0 \%)$ & \\
\hline Sleep difficu & & & & \\
\hline No & $116(72 \%)$ & $49(67 \%)$ & $58(67 \%)$ & \\
\hline Partially & $12(7.5 \%)$ & $3(4.1 \%)$ & $7(8.1 \%)$ & 0.759 \\
\hline Yes & $32(20 \%)$ & $21(29 \%)$ & $21(24 \%)$ & \\
\hline
\end{tabular}

${ }^{1} \mathrm{n}(\%) ;{ }^{2}$ Pearson's Chi-squared test; Fisher's exact test. All $p$-values were adjusted by the BenjaminiHochberg method. 
Table 5

Behavioral symptoms of family caregivers due to social isolation

\begin{tabular}{|c|c|c|c|c|c|}
\hline & $\begin{array}{l}\text { Argentina } \\
(N=108)\end{array}$ & $\begin{array}{c}\text { Brazil } \\
(N=111)\end{array}$ & $\begin{array}{c}\text { Chile } \\
(N=102)\end{array}$ & $\begin{array}{c}\text { Overall } \\
(N=321)\end{array}$ & Adjusted $p$ \\
\hline \multicolumn{6}{|l|}{ More tired } \\
\hline No & $40(37.0 \%)$ & $51(45.9 \%)$ & $31(30.4 \%)$ & $122(38.0 \%)$ & \multirow[t]{3}{*}{0.016} \\
\hline Partially & $20(18.5 \%)$ & $5(4.5 \%)$ & $9(8.8 \%)$ & $34(10.6 \%)$ & \\
\hline Yes & $47(43.5 \%)$ & $55(49.5 \%)$ & $59(57.8 \%)$ & $161(50.2 \%)$ & \\
\hline \multicolumn{6}{|c|}{ Increased burden } \\
\hline No & $45(41.7 \%)$ & $36(32.4 \%)$ & $25(24.5 \%)$ & $106(33.0 \%)$ & \multirow[t]{3}{*}{0.032} \\
\hline Partially & $14(13.0 \%)$ & $3(2.7 \%)$ & $9(8.8 \%)$ & $26(8.1 \%)$ & \\
\hline Yes & $46(42.6 \%)$ & $72(64.9 \%)$ & $64(62.7 \%)$ & $182(56.7 \%)$ & \\
\hline \multicolumn{6}{|c|}{ Worse health } \\
\hline No & $68(63.0 \%)$ & $59(53.2 \%)$ & $40(39.2 \%)$ & $167(52.0 \%)$ & \multirow[t]{3}{*}{$<0.001$} \\
\hline Partially & $23(21.3 \%)$ & $1(0.9 \%)$ & $10(9.8 \%)$ & $34(10.6 \%)$ & \\
\hline Yes & $13(12.0 \%)$ & $51(45.9 \%)$ & $48(47.1 \%)$ & $112(34.9 \%)$ & \\
\hline \multicolumn{6}{|c|}{ Greater physical effort } \\
\hline No & $60(55.6 \%)$ & $65(58.6 \%)$ & $28(27.5 \%)$ & $153(47.7 \%)$ & \multirow[t]{3}{*}{$<0.001$} \\
\hline Partially & $13(12.0 \%)$ & $2(1.8 \%)$ & $6(5.9 \%)$ & $21(6.5 \%)$ & \\
\hline Yes & $33(30.6 \%)$ & $44(39.6 \%)$ & $64(62.7 \%)$ & $141(43.9 \%)$ & \\
\hline \multicolumn{6}{|c|}{ More time spent with care } \\
\hline No & $58(53.7 \%)$ & $28(25.2 \%)$ & $16(15.7 \%)$ & $102(31.8 \%)$ & \multirow[t]{3}{*}{$<0.001$} \\
\hline Partially & $11(10.2 \%)$ & $5(4.5 \%)$ & $7(6.9 \%)$ & $23(7.2 \%)$ & \\
\hline Yes & $40(36.1 \%)$ & $78(70.3 \%)$ & $79(77.4 \%)$ & $195(61 \%)$ & \\
\hline \multicolumn{6}{|c|}{ Getting help from others } \\
\hline No & $49(45.4 \%)$ & $45(40.5 \%)$ & $58(56.9 \%)$ & $152(47.4 \%)$ & \multirow[t]{3}{*}{0.001} \\
\hline Partially & $21(19.4 \%)$ & $6(5.4 \%)$ & $7(6.9 \%)$ & $34(10.6 \%)$ & \\
\hline Yes & $35(32.4 \%)$ & $60(54.1 \%)$ & $34(33.3 \%)$ & $129(40.2 \%)$ & \\
\hline \multicolumn{6}{|l|}{ Loneliness } \\
\hline No & $64(59.3 \%)$ & $71(64.0 \%)$ & $46(45.1 \%)$ & $181(56.4 \%)$ & \multirow[t]{3}{*}{0.009} \\
\hline Partially & $17(15.7 \%)$ & $6(5.4 \%)$ & $7(6.9 \%)$ & $30(9.3 \%)$ & \\
\hline Yes & $25(23.1 \%)$ & $34(30.6 \%)$ & $46(45.1 \%)$ & $105(32.7 \%)$ & \\
\hline \multicolumn{6}{|c|}{ More nervous } \\
\hline No & $41(38.0 \%)$ & $52(46.8 \%)$ & $24(23.5 \%)$ & $117(36.4 \%)$ & \multirow[t]{3}{*}{$<0.001$} \\
\hline Partially & $33(30.6 \%)$ & $12(10.8 \%)$ & $11(10.8 \%)$ & $56(17.4 \%)$ & \\
\hline Yes & $32(29.6 \%)$ & $47(42.3 \%)$ & $64(62.7 \%)$ & $143(44.5 \%)$ & \\
\hline \multicolumn{6}{|l|}{ Sadder } \\
\hline No & $70(64.8 \%)$ & $54(48.6 \%)$ & $31(30.4 \%)$ & $155(48.3 \%)$ & \multirow[t]{3}{*}{$<0.001$} \\
\hline Partially & $16(14.8 \%)$ & $4(3.6 \%)$ & $16(15.7 \%)$ & $36(11.2 \%)$ & \\
\hline Yes & $20(18.5 \%)$ & $52(46.8 \%)$ & $52(51.0 \%)$ & $124(38.6 \%)$ & \\
\hline \multicolumn{6}{|c|}{ Sleep difficulties } \\
\hline No & $71(65.7 \%)$ & $71(64.0 \%)$ & $41(40.2 \%)$ & $183(57.0 \%)$ & \multirow[t]{3}{*}{$<0.001$} \\
\hline Partially & $17(15.7 \%)$ & $1(0.9 \%)$ & $7(6.9 \%)$ & $25(7.8 \%)$ & \\
\hline Yes & $17(15.7 \%)$ & $39(35.1 \%)$ & $51(50.0 \%)$ & $107(33.3 \%)$ & \\
\hline \multicolumn{6}{|c|}{ More irritability } \\
\hline No & $61(56.5 \%)$ & $59(53.2 \%)$ & $30(29.4 \%)$ & $150(46.7 \%)$ & \multirow[t]{3}{*}{$<0.001$} \\
\hline Partially & $20(18.5 \%)$ & $9(8.1 \%)$ & $16(15.7 \%)$ & $45(14.0 \%)$ & \\
\hline Yes & $23(21.3 \%)$ & $43(38.7 \%)$ & $53(52.0 \%)$ & $119(37.1 \%)$ & \\
\hline
\end{tabular}

Compulsive-obsessive behavior, hallucinations, increased forgetfulness, altered appetite, and increased difficulty in activities of daily living were reported more frequently among subjects with moderate to severe dementia (Table 4).

No significant differences were found in relation to the impact of social isolation on people with dementia considering the different socioeconomic levels.
Impact of social isolation on family caregivers of individuals with dementia

Family caregivers also experienced major changes caused by social isolation. Most caregivers (50.2\%) reported feeling more tired; in addition, $56.7 \%$ felt overwhelmed during this period and $34.9 \%$ reported having health problems (Table 5). Caregivers also 
Table 6

Behavioral symptoms of family caregivers due to social isolation according to severity of dementia

\begin{tabular}{|c|c|c|c|c|}
\hline & \multicolumn{3}{|c|}{ Dementia severity } & \multirow[t]{2}{*}{ Adjusted $l$} \\
\hline & $\begin{array}{c}\text { Mild } \\
N=161^{1}\end{array}$ & $\begin{array}{c}\text { Moderate } \\
N=74^{1}\end{array}$ & $\begin{array}{c}\text { Severe } \\
N=86^{1}\end{array}$ & \\
\hline \multicolumn{5}{|l|}{ More tired } \\
\hline No & $105(65 \%)$ & $43(58 \%)$ & $55(65 \%)$ & \multirow{3}{*}{0.262} \\
\hline Partially & $23(14 \%)$ & $8(11 \%)$ & $4(4.7 \%)$ & \\
\hline Yes & $33(20 \%)$ & $23(31 \%)$ & $26(31 \%)$ & \\
\hline \multicolumn{5}{|c|}{ Increased burden } \\
\hline No & $62(40 \%)$ & $22(30 \%)$ & $22(26 \%)$ & \multirow{3}{*}{0.018} \\
\hline Partially & $18(12 \%)$ & $5(6.8 \%)$ & $3(3.6 \%)$ & \\
\hline Yes & $76(49 \%)$ & $47(64 \%)$ & $59(70 \%)$ & \\
\hline \multicolumn{5}{|l|}{ Worse health } \\
\hline No & $97(62 \%)$ & $34(47 \%)$ & $36(43 \%)$ & \multirow{3}{*}{$<0.001$} \\
\hline Partially & $26(17 \%)$ & $6(8.2 \%)$ & $2(2.4 \%)$ & \\
\hline Yes & $33(21 \%)$ & $33(45 \%)$ & $46(55 \%)$ & \\
\hline \multicolumn{5}{|c|}{ Greater physical effort } \\
\hline No & $90(57 \%)$ & $32(44 \%)$ & $31(37 \%)$ & \multirow{3}{*}{0.008} \\
\hline Partially & $15(9.5 \%)$ & $3(4.1 \%)$ & $3(3.6 \%)$ & \\
\hline Yes & $53(34 \%)$ & $38(52 \%)$ & $50(60 \%)$ & \\
\hline \multicolumn{5}{|c|}{ More time spent with care } \\
\hline No & $68(43 \%)$ & $16(22 \%)$ & $18(22 \%)$ & \multirow{3}{*}{$<0.001$} \\
\hline Partially & $17(11 \%)$ & $2(2.7 \%)$ & $4(4.8 \%)$ & \\
\hline Yes & $73(46 \%)$ & $55(75 \%)$ & $61(73 \%)$ & \\
\hline \multicolumn{5}{|c|}{ Getting help from others } \\
\hline No & $82(52 \%)$ & $34(46 \%)$ & $36(43 \%)$ & \multirow{3}{*}{0.267} \\
\hline Partially & $19(12 \%)$ & $6(8.1 \%)$ & $9(11 \%)$ & \\
\hline Yes & $56(36 \%)$ & $34(46 \%)$ & $39(46 \%)$ & \\
\hline \multicolumn{5}{|l|}{ Loneliness } \\
\hline No & $97(61 \%)$ & $40(54 \%)$ & $44(52 \%)$ & \multirow{3}{*}{0.037} \\
\hline Partially & $21(13 \%)$ & $3(4.1 \%)$ & $6(7.1 \%)$ & \\
\hline Yes & $40(25 \%)$ & $31(42 \%)$ & $34(40 \%)$ & \\
\hline \multicolumn{5}{|c|}{ More nervous } \\
\hline No & $58(37 \%)$ & $25(34 \%)$ & $34(40 \%)$ & \multirow{3}{*}{$<0.001$} \\
\hline Partially & $46(29 \%)$ & $4(5.4 \%)$ & $6(7.1 \%)$ & \\
\hline Yes & $54(34 \%)$ & $45(61 \%)$ & $44(52 \%)$ & \\
\hline \multicolumn{5}{|l|}{ Sadder } \\
\hline No & $92(58 \%)$ & $31(42 \%)$ & $32(39 \%)$ & \multirow{3}{*}{0.008} \\
\hline Partially & $21(13 \%)$ & $7(9.5 \%)$ & $8(9.6 \%)$ & \\
\hline Yes & $45(28 \%)$ & $36(49 \%)$ & $43(52 \%)$ & \\
\hline \multicolumn{5}{|c|}{ Sleep difficulty } \\
\hline No & $102(65 \%)$ & $34(46 \%)$ & $47(56 \%)$ & \multirow{3}{*}{0.007} \\
\hline Partially & $17(11 \%)$ & $5(6.8 \%)$ & $3(3.6 \%)$ & \\
\hline Yes & $38(24 \%)$ & $35(47 \%)$ & $34(40 \%)$ & \\
\hline \multicolumn{5}{|c|}{ More irritability } \\
\hline No & $84(54 \%)$ & $32(44 \%)$ & $34(40 \%)$ & \multirow{3}{*}{0.007} \\
\hline Partially & $24(15 \%)$ & $7(9.6 \%)$ & $14(17 \%)$ & \\
\hline Yes & $49(31 \%)$ & $34(47 \%)$ & $36(43 \%)$ & \\
\hline
\end{tabular}

experienced behavioral changes during social isolation: $44.5 \%$ of them felt more nervous and more than a third $(38.6 \%)$ felt sadder or reported greater irritability (37.1\%). Moreover, $33.3 \%$ said that they had more difficulty in sleeping (Table 5).

These symptoms were also influenced by the severity of dementia. The caregivers of people with severe dementia experienced a greater burden $(70.0 \%)$ than did those who cared for people with mild dementia
(49.0\%). In addition, they reported suffering worsening of general health and having to exert greater physical effort in caring for these individuals with severe dementia during this period of social distancing (Table 6).

In addition, the family caregivers of individuals with moderate to severe dementia reported feeling more alone and sadder, having more sleep disorders and spending more time caring for these individuals, 
compared with the caregivers of individuals diagnosed with mild dementia (Table 6). The impact of social isolation on the family caregivers was not influence by socioeconomic level.

\section{DISCUSSION}

The present study investigated the impact of social isolation due to the SARS-CoV-2 pandemic on individuals with dementia and their family caregivers. The findings, although based on the perceptions of caregivers, suggest that both for people with dementia and their caregivers, the pandemic and social distancing have had a major effect and have triggered a series of behavioral, emotional, and physical consequences. To date, we are not aware of any other study that has assessed the impact of social isolation using data from three South American countries.

More than half of our sample of patients showed worsening of memory, according to the caregivers' reports, especially among individuals with moderate to severe dementia. Other studies have already shown that older people who were more socially isolated also had greater memory impairment and have suggested that the association between social isolation and memory decline is driven by the effect of social isolation on memory, and not the other way around [12-14].

Most individuals with dementia already have difficulties in remembering routine procedures and understanding the environment around them, as well as remembering the hygiene and self-care procedures needed at this stage [15]. Hence, the cognitive worsening observed in our sample should serve as an alert for all professionals involved with care of these individuals.

Closer monitoring and assessment of possible needs that arise during this period are essential, given that greater impairment of memory can further increase these individuals' risk of becoming infected with SARS-CoV-2. This is precisely because they do not remember and understand the important procedures that need to be followed in order to avoid this exposure [8]. In addition, this impairment of memory can compromise correct use of medication among individuals who have less cognitive impairment and are still able to organize and administer their own medication [9].

Several behavioral and psychological changes due to deprivation or reduced social contact during the pandemic period have also been reported, such as increased anxiety, agitation, and depression [16-18]. However, few studies have addressed these impacts on the population with dementia [19-21]. A recent study conducted in Italy evaluated neuropsychiatric changes among individuals with dementia and their caregivers during the lockdown period and found that there had been large increases in complaints of these symptoms in about two-thirds of both the patients and the caregivers [22].

We also found changes in anxiety symptoms and increased sadness and sleep disturbances in about one third of the people with dementia, which has also been found in previous studies. This confirms that this period can lead to various feelings of distress and symptoms of stress among these subjects. Our study also showed that the increase in anxiety was greater among individuals with mild to moderate dementia and that the symptoms of agitation worsened in those with severe dementia. Other studies have also shown that the absence of contact with other people and the restriction to home contribute to increased appearance of neuropsychiatric disorders and, in addition, increase the risk of morbidity and mortality [23, 24].

The functional performance of the people with dementia in our study sample also suffered notable impairment. Increased inactivity and reduced activity levels due to social isolation have also been found in other studies $[25,26]$. During this isolation period, most cognitive and physical stimulation programs, such as physiotherapy, occupational therapy, and other individual and collective activities, were totally or partially interrupted, which may have contributed to functional impairment $[8,9,20,27]$.

In relation to the family caregivers, we found that there had been increases in the frequency of symptoms of tiredness, sadness, irritability, and nervousness and that their workload had increased, such that they were spending more time on care. This was especially the case for caregivers of people with moderate and severe dementia. Previous studies reported that these caregivers were already suffering overload relating to emotional stress, sleep disorders, and higher risk of mental disorders [28-30]. Our study suggests that this burden may have been even greater during this period and was also influenced by the severity of dementia, thus causing major consequences regarding the physical and emotional state of caregivers.

Assessment of the diverse needs of family caregivers and guidance for them should be widely disseminated, in order to improve management at home and to alleviate the workload and stress [31]. As 
recommended by Alzheimer's Disease International, support for people living with dementia and their caregivers is paramount. In addition to physical protection against becoming infected with the virus, psychosocial, physical, and mental support needs to be offered to these individuals [32].

The present study had some limitations. It included a convenience sample, and, in addition, the interviews had to be carried out by telephone, since face-to-face meetings were not allowed, due to the pandemic situation. The interviews may have been influenced by the emotional state of each caregiver on the day of the survey. Moreover, the cross-sectional design of the study made it impossible to know the effects of isolation over time. Another limitation was that the study was carried out in three countries that were experiencing different moments of the pandemic. Despite this, social isolation was recommended in all three countries at the time of the survey. Finally, the FAST has been proposed as an instrument to assess severity of dementia in Alzheimer's disease and in the present study only dementia syndrome was considered, without further analyzes related to etiology.

Social isolation during the COVID-19 pandemic is causing significant impairment of memory, behavior, and activities of daily living among people with dementia. Management of these problems may have become even more difficult during this period, since there was discontinuity of care for most individuals. In addition, the prognosis for these individuals' dementia also needs to be evaluated or re-evaluated, because we do not know whether these changes will be reversible. Our research also showed that family caregivers' workload and emotional stress increased significantly.

Adaptation of the services that offer support for people with dementia and their caregivers is extremely necessary, especially during this period. Specific assessment of the effects caused by social distancing on an individual basis is essential, along with planning that includes the entire multidisciplinary health team involved with the care of these individuals [33].

Communication technologies can benefit and facilitate the approach to these people, although not all of them have access to this resource and some do not have the minimum ability to use them. Virtual consultations and use of cameras can be useful tools for monitoring and clinical management, with the aim of mitigating the impact of social isolation without exposing these people to an increased risk of SARSCoV-2 infection [34].
Further studies within this older adult population are needed, to elucidate the effects caused by social isolation due to the COVID-19 pandemic and to propose strategies for other similar periods that may arise in the future. In addition, it is necessary to create strategies for the return of these individuals to their usual activities whenever possible, and for their healthcare follow-up, so that their personal needs can be better faced.

\section{ACKNOWLEDGMENTS}

The authors would like to express their gratitude to the people with dementia and their family members who participated in this study. We would also like to thank the managers and employees of the outpatient clinics in the three countries that contributed to this research.

AS and DT received funding from ANID/ FONDAP/ 15150012. AS was partially supported by the Multi-partner consortium to expand dementia research in Latin America (ReDLat) which is supported by grants from the National Institutes of Health (R01AG057234), Alzheimer's Association (SG-20725707), Rainwater Charitable Foundation, and The Global Brain Health Institute. The content is solely the responsibility of the authors and does not represent the official views of these institutions. CM-C received funding from ANID/FONDECYT/1191726 and ANID - Millennium Science Initiative /Millennium Institute for Research on Depression and Personality-MIDAP ICS13_005. PC holds a senior researcher scholarship by CNPq, Brazil (bolsa de produtividade em pesquisa).

Authors' disclosures available online (https:// www.j-alz.com/manuscript-disclosures/20-1580r1).

\section{REFERENCES}

[1] World Health Organization (2021) COVID-19. https:// www.who.int/emergencies/diseases/novel-coronavirus2019, Accessed on February 6, 2021.

[2] Hellewell J, Abbott S, Gimma A, Bosse NI, Jarvis CI, Russell TW, Munday J, Kucharski AJ, Edmunds WJ, Centre for the Mathematical Modelling of Infectious Diseases COVID19 Working Group; Funk S, Eggo RM (2020) Feasibility of controlling COVID-19 outbreaks by isolation of cases and contacts. Lancet Glob Health 8, 488-496.

[3] Armitage R, Nellums LB (2020) COVID-19 and the consequences of isolating the elderly. Lancet Public Health 5, 256.

[4] Gerst-Emerson K, Jayawardhana J (2015) Loneliness as a public health issue: The impact of loneliness on health care utilization among older adults. Am J Public Health 105, 1013-1019. 
[5] Santini Z, Jose P, Cornwell E, Koyanagi A, Nielsen L, Hinrichsen C, Meilstrup C, Madsen K, Koushede V (2020) Social disconnectedness, perceived isolation, and symptoms of depression and anxiety among older Americans (NSHAP): A longitudinal mediation analysis. Lancet Public Health 5, 62-70.

[6] Wu J, Hasselgren C, Zettergren A, Zetterberg H, Blennow K, Skoog I, Halleröd B (2020) Impact of social networks and APOE $\varepsilon 4$ on dementia among older adults: Tests of possible interactions. Aging Ment Health 24, 395-404.

[7] Fernández S, Crivelli L, Guimet M, Allegri F, Pedreira E (2020) Psychological distress associated with COVID-19 quarantine: Latent profile analysis, outcome prediction and mediation analysis. $J$ Affect Disord 277, 75-84.

[8] Wang H, Li T, Barbarino P, Gauthier S, Brodaty H, Molinuevo JL, Xie H, Sun Y, Yu E, Tang Y, Weidner W, Yu X (2020) Dementia care during COVID-19. Lancet 395, 1190-1191.

[9] Brown EE, Kumar S, Rajji TK, Pollock BG, Mulsant BH (2020) Anticipating and mitigating the impact of the COVID-19 pandemic on Alzheimer's disease and related dementias. Am J Geriatr Psychiatry 28, 712-721.

[10] Reisberg B (1988) Functional assessment staging (FAST). Psychopharmacol Bull 24, 653-659.

[11] Associação Brasileira de Empresas de Pesquisa (ABEP). http://www.abep.org/criterio-brasil, Last updated September 9, 2019, Accessed on November 30, 2020.

[12] Read S, Comas-Herrera A, Grundy E (2020) Social isolation and memory decline in later-life. J Gerontol B Psychol Sci Soc Sci 75, 367-376.

[13] DiNapoli EA, Wu B, Scogin F (2014) Social isolation and cognitive function in Appalachian older adults. Res Aging 36, 161-179.

[14] Shankar A, Hamer M, McMunn A, Steptoe A (2013) Social isolation and loneliness: Relationships with cognitive function during 4 years of follow-up in the English Longitudinal Study of Ageing. Psychosom Med 75, 161-170.

[15] McMichael TM, Currie DW, Clark S, Pogosjans S, Kay M, Schwartz NG,Lewis J, Baer A, Kawakami V,Lukoff MD, Ferro J, Brostrom-Smith C, Rea TD, Sayre MR, Riedo FX, Russell D, Hiatt B, Montgomery P, Rao AK, Chow EJ, Tobolowsky F, Hughes MJ, Bardossy AC, Oakley LP, Jacobs JR, Stone ND, Reddy S, Jernigan JA, Honein MA, Clark TA, Duchin JS (2020) Epidemiology of COVID-19 in a long-term care facility in King County, Washington. $N$ Engl J Med 382, 2005-2011.

[16] Ho CS, Chee CY, Ho RC (2020) Mental health strategies to combat the psychological impact of COVID-19 beyond paranoia and panic. Ann Acad Med Singapore 49, 155-160.

[17] Serafini G, Parmigiani B, Amerio A, Aguglia A, Sher L, Amore M (2020) The psychological impact of COVID-19 on the mental health in the general population. QJM 113, 531-537.

[18] Salari N, Hosseinian-Far A, Jalali R, Vaisi-Raygani A, Rasoulpoor S, Mohammadi M, Shabnam R, Khaledi-Paveh B (2020) Prevalence of stress, anxiety, depression among the general population during the COVID-19 pandemic: A systematic review and meta-analysis. Global Health 16, 57.

[19] Sorbara M, Graviotto HG, Lage-Ruiz GM, TurizoRodriguez C, Sotelo-López L, Serra A, Gagliardi C, Heinemann G, Martinez P, Ces-Magliano F, Serrano C
(2020) COVID-19 and the forgotten pandemic: Follow-up of neurocognitive disorders during lockdown in Argentina. Neurologia 36, 9-15.

[20] Cohen G, Russo MJ, Campos JA, Allegri RF (2020) COVID-19 epidemic in Argentina: Worsening of behavioral symptoms in elderly subjects with dementia living in the community. Front Psychiatry 11, 866.

[21] Lara B, Carnes A, Dakterzada F, Benitez I, Piñol-Ripoll G (2020) Neuropsychiatric symptoms and quality of life in Spanish patients with Alzheimer's disease during the COVID-19 lockdown. Eur J Neurol 27, 1744-1747.

[22] Cagnin A, Di Lorenzo R, Marra C, Bonanni L, Cupidi C, Laganà V, Rubino E, Vacca $\mathrm{A}$, Provero $\mathrm{P}$, Isella V, Vanacore N, Agosta F, Appollonio I, Caffarra P, Pettenuzzo I, Sambati R, Quaranta D, Guglielmi V, Logroscino G, Filippi M, Tedeschi G, Ferrarese C, Rainero I, Bruni A, SINdem COVID-19 Study Group (2020) Behavioral and psychological effects of coronavirus disease-19 quarantine in patients with dementia. Front Psychiatry 11, 578015.

[23] Friedler B, Crapser J, McCullough L (2015) One is the deadliest number: The detrimental effects of social isolation on cerebrovascular diseases and cognition. Acta Neuropathol 129, 493-509.

[24] Taylor H, Taylor R, Nguyen A, Chatters L (2018) Social isolation, depression, and psychological distress among older adults. J Aging Health 30, 229-246.

[25] Schrempft S, Jackowska M, Hamer M, Steptoe A (2019) Associations between social isolation, loneliness, and objective physical activity in older men and women. BMC Public Health 19, 74.

[26] Kobayashi LC, Steptoe A (2018) Social isolation, loneliness, and health behaviors at older ages: Longitudinal cohort study. Ann Behav Med 52, 582-593.

[27] Bostanciklioglu M (2020) Severe acute respiratory syndrome coronavirus 2 is penetrating to dementia research. Curr Neurovasc Res 17, 342-343.

[28] Cheng ST (2017) Dementia caregiver burden: A research update and critical analysis. Curr Psychiatry Rep 19, 4.

[29] Kamiya M, Sakurai T, Ogama N, Maki Y, Toba K (2014) Factors associated with increased caregivers' burden in several cognitive stages of Alzheimer's disease. Geriatr Gerontol Int 2, 45-55

[30] Chiao CY, Wu HS, Hsiao CY (2015) Caregiver burden for informal caregivers of patients with dementia: A systematic review. Int Nurs Rev 62, 340-350.

[31] Alzheimer Europe. COVID-19 - living with dementia. https://www.alzheimereurope.org/Living-with-dementia/ COVID-19, Last updated June 3, 2020, Accessed on October 2, 2020.

[32] Alzheimer's Disease International ADI offers advice and support during COVID-19. https://www.alz.co.uk/ news/adi-offers-advice-and-support-during-covid-19, Last updated March 23, 2020, Accessed on October 8, 2020.

[33] Brooks SK, Webster RK, Smith LE, Woodland L, Wessely S, Greenberg N, Rubin G (2020) The psychological impact of quarantine and how to reduce it: Rapid review of the evidence. Lancet 395, 912-920.

[34] Cuffaro L, Di Lorenzo F, Bonavita S, Tedeschi G, Leocani L, Lavorgna L (2020) Dementia care and COVID-19 pandemic: A necessary digital revolution. Neurol Sci 41, 1977-1979. 